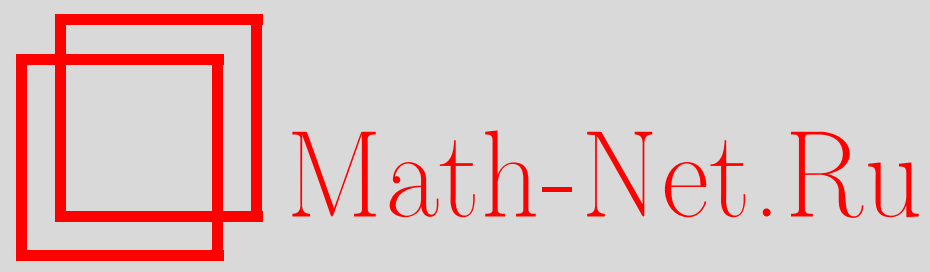

B. А. Артамонов, Периодические модули над общими квантовыми лорановскими полиномами, Матем. заметки, 1997, том 61, выпуск 1, 10-17

DOI: https://doi.org/10.4213/mzm1477

Использование Общероссийского математического портала Math-Net.Ru подразумевает, что вы прочитали и согласны с пользовательским соглашением http://www.mathnet.ru/rus/agreement

Параметры загрузки:

IP : 54.198 .64 .247

26 апреля 2023 г., 14:37:07

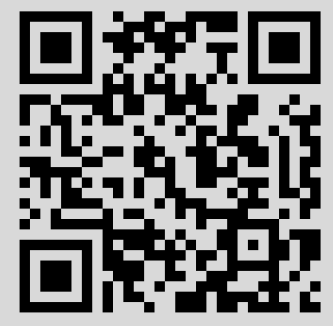




\title{
ПЕРИОДИЧЕСКИЕ МОДУЛИ НАД ОБЩИМИ КВАНТОВЫМИ ЛОРАНОВСКИМИ ПОЛИНОМАМИ
}

\section{В. А. Артамонов}

\begin{abstract}
Указывается индукцией по числу переменных конструкция периодических конечнопорожденных модулей над общими квантовьми лорановскими многочленами.

Библиографоия: 12 названий.
\end{abstract}

Настоящая работа является продолжением исследований строения конечнопорожденных модулей над обшими квантовыми лорановскими полиномами, начатых в [1], [2]. В [1] показано, что конечнопорожденные проективные модули ранга не меньше двух над алгеброй общих квантовых лорановских полиномов свободны. В [2] показано, что конечнопорожденные модули над общими квантовыми лорановскими полиномами разлагаются в прямую сумму периодической части, являющейся циклическим подмодулем, и конечнопорожденного проективного подмодуля. В настоящей работе доказано, что если $M$ - конечнопорожденньй периодический модуль над алгеброй общих квантовых лорановских полиномов, то можно выбрать такие переменные $X_{1}, \ldots, X_{n}$, что $M$ является конечнопорожденным проективным модулем над подалгеброй, порождаемой $X_{1}, \ldots, X_{n-1}$ (см. теорему 2.4 ). Показано, что верно и обратное утверждение.

Из работ, относяшихся к этой теме, можно отметить книгу [3] и работы [4]-[6], а также другие работы этих авторов последних лет.

Отметим, что квантовые полиномы играют важную роль в построении некоммутативной геометрии [7].

1. Основные конструкции. Пусть $D$ - тело над центральньм подполем $k$ и в $D$ заданы $k$-автоморфизмы $\alpha_{1}, \ldots, \alpha_{n}$, где $n \geqslant 2$. Предположим, что заданы элементы $q_{i j} \in D^{*}, i, j=1, \ldots, n$, причем $q_{i i}=q_{i j} q_{j i}=Q_{i j r} Q_{j r i} Q_{r i j}=1$, где $Q_{i j r}=q_{i j} \alpha_{j}\left(q_{i r}\right)$. Рассмотрим ассоциативную $k$-алгебру, порожденную элементами тела $D$, элементами $X_{1}, \ldots, X_{n}, X_{1}^{-1}, \ldots, X_{n}^{-1}$ и заданную определяющими соотношениями

$$
\begin{gathered}
X_{i} X_{i}^{-1}=X_{i}^{-1} X_{i}=1, \quad i=1, \ldots, n, \\
X_{i} z=\alpha_{i}(z) X_{i}, \quad z \in D, \quad i=1, \ldots, n, \\
X_{i} X_{j}=q_{i j} X_{j} X_{i}, \quad i, j=1, \ldots, n .
\end{gathered}
$$

Работа выполнена при частичной поддержке Российского фонда фундаментальных исследований, грант № 96-01-00627, фонда INTAS, грант № 93-2618, и Международного научного фонда, грант JBX100. 
Эта алгебра является скрешенным произведением $A=D \sharp_{t} k G$, где $G$ - свободная абелева группа с базой $X_{1}, \ldots, X_{n}, t: k G \otimes k G \rightarrow D^{*}$ - некоторьй 2-коцикл, причем $t\left(X_{i}, X_{j}\right) t\left(X_{j}, X_{i}\right)^{-1}=q_{i j}[1],[8],[3]$. Кроме того, имеется слабое действие $G$ на $D$, причем

$$
X_{i} \circ z=\alpha_{i}(z), \quad z \in D, \quad i=1, \ldots, n .
$$

Таким образом, $A$ является свободным левым и правым $D$-модулем, базу которого составляют одночлены $X_{1}^{m_{1}} \cdots X_{n}^{m_{n}}, m_{i} \in \mathbb{Z}$.

Пусть $N$ - подгруппа в мультипликативной групе $D^{*}$ тела $D$, порожденная коммутантом $\left[D^{*}, D^{*}\right]$ и всеми элементами вида $z^{-1} \alpha_{i}(z), z \in D^{*}, i=1, \ldots, n$.

Теорема 1.1. Пусть $H$ - подгруппа в $D^{*} / N$, порожсенная образами всех әлементов $t(g, h) t(h, g)^{-1}$, где $g, h \in G$. Тогда $H$ порохдается образами әлементов $q_{i j}, 1 \leqslant i<j \leqslant n$.

ДоказАтЕльство. Так как $A=D \sharp_{t} k G$, то в силу (1) (см. также [1], [8], [3]) получаем

$$
\begin{gathered}
g \circ(h \circ z)=t(g, h)(g h \circ z) t(g, h)^{-1}, \quad[g \circ t(h, u)] t(g, h u)=t(g, h) t(g h, u), \\
t\left(X_{i}, X_{j}\right)= \begin{cases}q_{i j}, & \text { если } i \leqslant j, \\
1, & \text { если } i \geqslant j,\end{cases}
\end{gathered}
$$

где $g, h, u \in G, z \in D$.

Из (2) вытекает, что для $g, h \in G, z \in D$ по модулю $N g \circ(h \circ z) \equiv(g h) \circ z$. Отсюда $g \circ z \equiv z \bmod N$, ибо $X_{i} \circ z \equiv z \bmod N$. Если $g, h \in G$, то в $D \sharp_{t} k G$ в силу определения скрещенного произведения $(1 \sharp g)(1 \sharp h)=t(g, h) \sharp g h$. В частности,

$$
\begin{gathered}
(1 \sharp g)(1 \sharp h)=t(g, h) t(g, h)^{-1}(1 \sharp h)(1 \sharp g), \\
t\left(X_{i}, X_{j}\right) t\left(X_{j}, X_{i}\right)^{-1}=q_{i j}, \quad 1 \leqslant i, j \leqslant n .
\end{gathered}
$$

Таким образом, в силу (1)-(3) получаем, как и в [4], что мультипликативная подгруппа $H$ в $D^{*} / N$, порождаемая образами $t(g, h) t(g, h)^{-1}, g, h \in G$, совпадает с подгруппой, порождаемой образами $q_{i j}, 1 \leqslant i<j \leqslant n$.

Всюду в дальнейшем мы будем предполагать, что $A$ является общей алгеброй квантовых лорановских полиномов, т.е. образы элементов $q_{i j}, 1 \leqslant i<j \leqslant n$, независимы в мультипликативной абелевой групе $D^{*} / N$. Это эквивалентно тому, что подгрупп $H$ в мультипликативной абелевой групе $D^{*} / N$ имеет максимально возможный ранг, равный $n(n-1) / 2$. В силу теоремы 1.1 определение общей алгебры лорановских полиномов не зависит от выбора базиса в $G$. Отметим, что пример алгебры общих квантовых лорановских полиномов получается, если, например, в качестве $D$ взять поле рациональных функций $k\left(q_{i j} \mid 1 \leqslant i<j \leqslant n\right)$ от неизвестньх $q_{i j}, 1 \leqslant i<j \leqslant n$, и в качестве автоморфизмов $\alpha_{1}, \ldots, \alpha_{n}$ взять тождественные автоморфизмы поля $k\left(q_{i j} \mid 1 \leqslant i<j \leqslant n\right)$.

В силу предложения 4 [1] и следствия из него, как и в [9], получаем, что в этом случае алгебра $A$ является простой наследственной областью размерности Крулля 1 . Пусть $F$ - тело частных алгебры $A$. Если $M-$ конечнопорожденньй $A$-модуль, то $р а н г о м$ $M$ назовем число $r(M)=\operatorname{dim}_{F}\left(F \otimes_{D} M\right)$. Периодической частью $t(M)$ модуля $M$ над $A$ назовем множество всех периодических элементов в $M$, т.е. множество всех таких элементов из $M$, аннулятор которых в $A$ отличен от нуля. Модуль $M$ периодический, если $M=t(M)$. В [2] доказана 
Теорема 1.2. Пусть $M$ - конечнопорожденный $A$-модуль ранга $r \geqslant 0$. Тогда $t(M)$ является подмодулем. Кроме того, $M=t(M) \oplus N$, где $t(M)$ - ииклический артинов $A$-модуль, $N$ - проективный $A$-модуль ранга $r$. Если $r=0$, то $N=0$. Если $r=1$, то модуль $N$ изоморфен левому идеалу в $A$. Если $r>1$, то модуль $N$ свободен.

Всюду в дальнейшем через $\left\langle g_{1}, \ldots, g_{r}\right\rangle$ будет обозначаться подалгебра, порожденная в $A=D \sharp_{t} k G$, порожденная $D$ и элементами $g_{1}^{ \pm 1}, \ldots, g_{r}^{ \pm 1} \in G$. Следующее утверждение фактически доказано в [10].

ПРЕДЛОЖЕНИЕ 1.3. Пусть $X_{1}, \ldots, X_{n}-$ база $G u f$ - ненулевой әлемент из $\left\langle X_{1}, \ldots, X_{r}\right\rangle, r \leqslant n$, причем $X_{r}$ входит в запись $f$. Тогда существуют такие натуральные числа $l_{1}, \ldots, l_{r-1}$, и базис $Y_{1}, \ldots, Y_{n}$ в $G$, что

$$
X_{i}= \begin{cases}Y_{i} Y_{r}^{l_{i}}, & \text { если } i<r \\ Y_{i}, & \text { если } i=r\end{cases}
$$

и в $f$ как в многочлене от $Y_{1}, \ldots, Y_{r}$ разные одночлены имеют разные показатели при $Y_{r}$.

ДокАЗАТЕЛЬСТво. Домножая $f$ на одночлен можно считать, что показатели при всех переменных $X_{i}$ неотрицательны. Пусть $p$ - простое число, большее всех показателей по $X_{1}, \ldots, X_{r}$ одночленов в $f$. Совершим замену переменных вида (4), где $l_{i}=p^{i}$. Получим, что каждьй одночлен из $f$ имеет вид

$$
\lambda X_{1}^{s_{1}} \cdots X_{r}^{s_{r}}=\lambda^{\prime} Y_{1}^{s_{1}} \cdots Y_{r-1}^{s_{r-1}} Y_{r}^{s_{r}+p s_{1}+\cdots+p^{r-1} s_{r-1}}
$$

где $\lambda, \lambda^{\prime} \in D^{*}$. Предположим, что $\left(t_{1}, \ldots, t_{r}\right)$ - другой набор показателей у одночлена из $f$, причем после той же замены переменных показатель при $Y_{r}$ стал равньм $s_{r}+p s_{1}+\cdots+p^{r-1} s_{r-1}$. В силу единственности $p$-адического разложения получаем, что $\left(t_{1}, \ldots, t_{r}\right)=\left(s_{1}, \ldots, s_{r}\right)$.

ПРЕДЛОЖЕНИЕ 1.4. Пусть $M$ - некоторый $A$-модуль, причем в $G$ существует такой базис $X_{1}, \ldots, X_{n}$, что $M$ является конечнопорохсденным $\left\langle X_{1}, \ldots, X_{n-2}\right\rangle$ модулем. Тогда $M=0$.

ДокАЗАТЕЛЬСтво. Модуль $M$ конечнопорожден над $A$. По теореме 1.2 имеем $M=t(M) \oplus N$, где $t(M)$ - периодическая часть $M$, а $N$ - конечнопорожденный проективньй $A$-модуль. Если $N \neq 0$, то модуль $N$ либо свободен, либо изоморфен левому идеалу в $A$. Во всех случаях $N$ содержит свободньй циклический $A$-подмодуль $U$. Так как алгебра $\left\langle X_{1}, \ldots, X_{n-2}\right\rangle$ нётерова слева, то $U$ является конечнопорожденным модулем над $\left\langle X_{1}, \ldots, X_{n-2}\right\rangle$, что неверно. Итак, $M=t(M)$, и потому модуль $M=A m$ является циклическим.

Пусть $j=n, n-1$. Так как $\left\langle X_{1}, \ldots, X_{n-2}\right\rangle$-модуль $M$ конечнопорожден, то он нётеров. Поэтому существует такое натуральное число $L$, что

$$
X_{j}^{ \pm(L+1)} m \in \sum_{i=-L}^{L}\left\langle X_{1}, \ldots, X_{n-2}\right\rangle X_{j}^{i} m .
$$


Положим $I=\operatorname{Ann}_{A} m$. Предположим сначала, что

$$
I \cap\left\langle X_{1}, \ldots, X_{n-2}\right\rangle \neq 0 .
$$

Пусть $f$ - ненулевой элемент из этого пересечения. Если $f \notin D$, то, например, $X_{n-2}$ входит в запись $f$. Совершив в силу предложения 1.3 замену $X_{1}, \ldots, X_{n} \rightsquigarrow Y_{1}, \ldots, Y_{n}$ переменных в $G$ c $r=n-2$ можно считать, что относительно $Y_{1}, \ldots, Y_{n-2}$ разные одночлены в $f$ имеют разные показатели при $Y_{n-2}$. В частности, старший и младший члены относительно $Y_{n-2}$ имеют соответственно вид

$$
a Y_{n-2}^{l}, \quad b Y_{n-2}^{m}, \quad l \leqslant m,
$$

где $a, b$ - одночлены от $Y_{1}, \ldots, Y_{n-3}$. В силу (5) получаем, что $M=A m \simeq A / I$ является конечнопорожденным $\left\langle Y_{1}, \ldots, Y_{n-3}\right\rangle$-модулем. Продолжая этот процесс, выберем в $G$ такой базис $Z_{1}, \ldots, Z_{n}$, что $M$ является конечнопорож денным $\left\langle Z_{1}, \ldots, Z_{d}\right\rangle$-модулем для некоторого $d \leqslant n-2$, причем $I \cap\left\langle Z_{1}, \ldots, Z_{d}\right\rangle=0$.

Как отмечено в [9] и [1], ненулевые элементы из $\left\langle Z_{1}, \ldots, Z_{d}\right\rangle$ удовлетворяют условию Оре в $A$. Пусть $B$ - локализация $A$ по этой системе. Тогда $B \otimes_{A}(A / I)$ является ненулевым конечномерным левым векторным пространством над телом частных $\left\langle Z_{1}, \ldots, Z_{d}\right\rangle$. По [9] и предложению 4 из [1] это невозможно.

2. Левые идеалы и модули. Пусть $I$ - ненулевой левый идеал в $A$ и $f \in I \backslash 0$. В соответствии с предложением 1.3 в $G$ существует такая система свободных образуюших $X_{1}, \ldots, X_{n}$, что разные одночлены $f$ имеют разные показатели при $X_{n}$. Таким образом, как и в предложении $1.4, A / I$ является конечнопорожденным $\left\langle X_{1}, \ldots, X_{n-1}\right\rangle$-модулем.

ПРЕДЛОЖЕНИЕ 2.1. Если $A \neq I$, mo

$$
I \cap\left\langle X_{1}, \ldots, X_{n-1}\right\rangle=0 .
$$

ДокАЗАТЕЛЬСтво. Предположим противное. Пусть $g$ - ненулевой элемент из $\left[I \cap\left\langle X_{1}, \ldots, X_{n-1}\right\rangle\right] \backslash D$. Как и в предложении 1.4 , совершая замену переменных, можно в $G$ выбрать такой базис $Y_{1}, \ldots, Y_{n}$, что $A / I$ является конечнопорожденным модулем над $\left\langle X_{1}, \ldots, X_{n-2}\right\rangle$. Но по предложению 1.4 это невозможно.

Предположим теперь, что $M$ - ненулевой периодический $A$-модуль. По теореме 1.2 модуль $M$ цикличен с образуюшим элементом $m$. Пусть $I=\operatorname{Ann}_{A} m \neq 0$ и $f \in I \backslash 0$. Выберем в соответствии с предложением 1.3 для $f$ и предложением 2.1 систему свободных образующих в $G$, которую мы обозначим $X_{1}, \ldots, X_{n}$. Тогда $M$ является конечнопорожденным $\left\langle X_{1}, \ldots, X_{n-1}\right\rangle$-модулем и вьполнено (6) По (6) элемент $m$ не является периодическим над $\left\langle X_{1}, \ldots, X_{n-1}\right\rangle$. Пусть $N$ - периодическая часть $M$ как модуля над $\left\langle X_{1}, \ldots, X_{n-1}\right\rangle$.

ПРЕДЛОЖЕНИЕ 2.2. $N$ является $A$-подмодулем в $M$.

ДокАЗАТЕЛЬСтво. Пусть $z_{1}, z_{2} \in N$ и $f_{1} z_{1}=f_{2} z_{2}=0$, где $f_{1}, f_{2} \in\left\langle X_{1}, \ldots, X_{n-2}\right\rangle \backslash 0$. Ненулевые элементы из $\left\langle X_{1}, \ldots, X_{n-1}\right\rangle$ удовлетворяют условию Оре в $A$. Поэтому существуют такие $h_{1}, h_{2} \in\left\langle X_{1}, \ldots, X_{n-1}\right\rangle \backslash 0$, что $h_{1} f_{1}=h_{2} f_{2}$. Отсюда $h_{1} f_{1}\left(z_{1}+z_{2}\right)=0$, т.e. $z_{1}+z_{2} \in N$.

Предположим, что $z \in N$ и $f z=0$ для некоторого $f \in\left\langle X_{1}, \ldots, X_{n-1}\right\rangle \backslash 0$. Если $g \in A \backslash 0$, то в силу условия Оре найдутся такие $f^{\prime} \in\left\langle X_{1}, \ldots, X_{n-1}\right\rangle \backslash 0, g^{\prime} \in A \backslash 0$, что $f^{\prime} g=g^{\prime} f$. Отсюда $f^{\prime} g z=g^{\prime}(f z)=0$ и $g z \in N$. 
ПРЕДЛОЖЕНИЕ 2.3. Если $N$ из предложения 2.2, mо $N=0$.

ДокАЗАТЕЛЬСтво. Пусть $N \neq 0$. В силу теоремы $1.2\left\langle X_{1}, \ldots, X_{n-1}\right\rangle$-модуль $N$ цикличен, т.е. $N \simeq\left\langle X_{1}, \ldots, X_{n-1}\right\rangle / J$, где $J$ - левый идеал в $\left\langle X_{1}, \ldots, X_{n-1}\right\rangle$. Как и выше, совершив замену переменных, можно считать, что $N$ является конечнопорожденным $\left\langle X_{1}, \ldots, X_{n-2}\right\rangle$-модулем. Остается воспользоваться предложением 1.4.

Теорема 2.4. Пусть $M$ - ненулевой периодический $A$-модуль. Тогда модуль $M$ иикличен и в $G$ существует такой базис $X_{1}, \ldots, X_{n}$, что $M$ является конечнопорожденным проективным $\left\langle X_{1}, \ldots, X_{n-1}\right\rangle$-модулем.

ДокАЗАТЕЛЬСТВо вытекает из теоремы 1.2 и предложения 2.3.

3. Примеры. Пусть $M, X_{1}, \ldots, X_{n}$ выбраны в соответствии с условиями теоремы 2.4. Положим $U=\left\langle X_{1}, \ldots, X_{n-1}\right\rangle$. Предположим, что $M$ как $U$-модуль свободен. В силу [1] это всегда так, если ранг $M$ как $U$-модуля не меньше двух. Выберем базис $\mathbf{e}=\left(e_{1}, \ldots, e_{r}\right)-$ базис $M$ как $U$-модуля. Тогда

$$
X_{n} e_{i}=\sum_{j} z_{i j} e_{j}, \quad z_{i j} \in U .
$$

Если $m \in M$, то

$$
m=\sum_{j=1}^{d} m_{i} e_{i}, \quad m_{i} \in U
$$

и поэтому

$$
Y_{n} m=\sum_{i, j=1}^{d} \alpha_{n}\left(m_{i}\right) a_{i j} e_{j},
$$

где $\alpha_{n}$ - продолжение на $U$ автоморфизма $\alpha_{n}$ на $D$, причем $\alpha_{n}\left(X_{j}\right)=q_{n j} X_{j}$. Так как элемент $X_{n}$ обратим в $A$, то $Z=\left(z_{i j}\right) \in \mathrm{GL}(r, U)$.

Обратно, если $M-$ свободньй $U$-модуль с базой $e_{1}, \ldots, e_{r}$ и $Z=\left(z_{i j}\right) \in \mathrm{GL}(r, U)$, то $M$ является $A$-модулем относительно умножения на $X_{n}$, задаваемого $(7)$. Действительно, нетрудно проверить, что для всех $m \in M, z \in D$

$$
X_{n}(z m)=\alpha_{n}(z)\left(X_{n} m\right), \quad X_{n}\left(X_{j} m\right)=\alpha_{n}\left(X_{j}\right)\left(X_{n} m\right) .
$$

Заметим, что построенный модуль $M$ является периодическим. Действительно, $A$-модуль $M$ конечнопорожден, ибо он конечнопорожден как $U$-модуль. Если бы он не был периодическим, то, как отмечалось вьше, $M$ содержал бы $A$-подмодуль $H$, изоморфньй свободному циклическому $A$-модулю. Но $H$ не является конечнопорожденным $U$-модулем. Получается противоречие с нётеровостью $U$.

Отметим, что если в $M$ как в $U$-модуле выбрать другой базис $\mathbf{e}^{\prime}$ с матрицей перехода $C$, где $\mathbf{e}^{\prime}=C \mathbf{e}$, то по (7) получаем, что матрища $Z$ заменится на матрицу $Z^{\prime}=\alpha_{n}(C) Z C^{-1}$.

Покажем теперь, что ранг модуля $M$ зависит от выбора свободных образующих $X_{1}, \ldots, X_{n}$. Приведем соответствующий пример. Пусть $D=k-$ поле, $n=2$ и $\alpha_{1}=\alpha_{2}$ - тождественньй автоморфизм поля $k$. Предположим, что $q \in k^{*}$. Рассмотрим алгебру $A=k\left[X^{ \pm 1}, Y^{ \pm 1}\right]$, где определяющее соотношение имеет вид $X Y=q Y X$. В качестве модуля $M$ рассмотрим циклический модуль $M=A / A f$, где $f=1+X Y+X^{2} Y$. 
ПРЕДЛОЖЕНИЕ 3.1. М не является конечнопорожденныц $\langle X\rangle$-модулем.

ДоКАЗАТЕЛЬСТво. Предположим противное, $M$ является конечнопорожденным $\langle X\rangle$-модулем. Так как модуль $M$ нётеров над $\langle X\rangle$, то существует такое натуральное число $m$, что в $A / A f$

$$
Y^{m}+A f=\sum_{i=0}^{m-1}\left(a_{i}(X) Y^{i}+A f\right)
$$

где $a_{i}(X) \in\langle X\rangle$. Но тогда

$$
Y^{m}-\sum_{i=0}^{m-1} a_{i}(X) Y^{i} \in A f .
$$

Без ограничения обшности, можно предполагать, что

$$
Y^{m}-\sum_{i=0}^{m-1} a_{i}(X) Y^{i}=\sum_{i=0}^{m-1} b_{i}(X) Y^{i}\left(1+X Y+X^{2} Y\right) .
$$

Сравнивая коэффициенты при $Y^{m}$ в обеих частях (8), получаем $1=b_{m-1}(X)\left(X+X^{2}\right)$, т.е. элемент $X+X^{2}$ обратим в $A$, что неверно.

ПРЕДЛОЖЕНИЕ 3.2. Пусть $p-$ простое нечетное число, $и X_{1}=X Y^{-p}$. Тогда $M$ является свободным $\left\langle X_{1}\right\rangle$-модулем ранга $1+2 p$.

ДокАЗАТЕльство. Для доказательства заметим, что $f=1+X_{1} Y^{1+p}+q^{p} X_{1}^{2} Y^{1+2 p}$ является унитарным многочленом от $Y$ степени $1+2 p$.

Пусть $M, U, A$ такие же, как и в начале этого раздела. Если $M-$ проективньй несвободный $U$-модуль, то по теореме $1.2 U$-модуль $M$ изоморфен левому идеалу в $U$.

ПредлоЖенИЕ 3.3. На любом ненулевом левом идеале I в $U$ существует структура $A$-модуля, продолжающая структуру $U$-модуля.

ДоКАЗАТЕЛЬСТво. Как отмечено в [2], ненулевой $U$-модуль $I$ порождается двумя элементами $f, g \neq 0$. Пусть $S$ - множество всех ненулевых элементов из $S$. Тогда $S^{-1} I$ совпадает с телом частных $U$. Поэтому найдутся такие $p, q \in I \backslash 0$, что

$$
\alpha_{n}(f) \alpha_{n}(g)^{-1}=p q^{-1} \text {. }
$$

Отсюда $\alpha_{n}(f)^{-1} p=\alpha_{n}(g)^{-1} q=u \in S^{-1} I$. Если $t \in I$, то $t=a f+b g$, где $a, b \in U$. При этом

$$
\alpha_{n}(a f) u+\alpha_{n}(b g) u=\alpha_{n}(a) \alpha_{n}(f) u+\alpha_{n}(b) \alpha_{n}(g) u=\alpha_{n}(a) p+\alpha_{n}(b) q \in I .
$$

Положим

$$
X_{n} t=\alpha_{n}(a) p+\alpha_{n}(b) q \in I .
$$

Для того, чтобы показать, что определение (10) корректно, достаточно проверить, что если $a f=b g$, то $\alpha_{n}(a) p=\alpha_{n}(b) q$. Действительно,

$$
\alpha_{n}(a) p=\alpha_{n}(a) \alpha_{n}(f) \alpha_{n}(g)^{-1} q=\alpha_{n}(a f) \alpha_{n}(g)^{-1} q=\alpha_{n}(b g) \alpha_{n}(g)^{-1} q=\alpha_{n}(b) q .
$$

Отметим ряд следствий из полученных утверждений. 
СЛЕДСТВИЕ 3.4. Пусть $M$ - конечнопорожденный периодический $A$-модуль $u$ $X_{1}, \ldots, X_{n}-$ база в $G$, причем $M$ является конечнопорожсденным проективным $\left\langle X_{1}, \ldots, X_{n-1}\right\rangle$-модулем. Предположим, что $N-A$-подмодуль в $M$. Тогда $N u$ $M / N$ являются конечнопорохсдеными проективными $\left\langle X_{1}, \ldots, X_{n-1}\right\rangle$-модулями. $B$ частности, если ранг $M$ как $\left\langle X_{1}, \ldots, X_{n-1}\right\rangle$-модуля равен 1 , то $M$ как $A$-модуль неприводим. Eсли ранг $M$ как $\left\langle X_{1}, \ldots, X_{n-1}\right\rangle$-модуля равен $r u$ әлементы $g_{1}, \ldots, g_{r} \in M$ независимы над $\left\langle X_{1}, \ldots, X_{n-1}\right\rangle$, то они порожсают М как A-модуль.

Для формулировки и доказательства последнего следствия нам потребуется

ПРЕДЛОЖЕНИЕ 3.5. Пусть в $\quad G \quad$ выбрана база $X_{1}, \ldots, X_{n} \quad u \quad N \quad$ - некоторый $\left\langle X_{2}, \ldots, X_{n}\right\rangle$-модуль, являющийся конечнопорожденным проективным $\left\langle X_{2}, \ldots, X_{n-1}\right\rangle$-модулем. Если $N^{\prime}$ - некоторьй $\left\langle X_{2}, \ldots, X_{n}\right\rangle$-подмодуль в $N$, то естественный морфизм $A$-модулей

$$
A \otimes_{\left\langle X_{2}, \ldots, X_{n}\right\rangle} N^{\prime} \rightarrow A \otimes_{\left\langle X_{2}, \ldots, X_{n}\right\rangle} N
$$

ингективен.

ДокАЗАтЕльство. Необходимо лишь заметить, что $A$ является проективным и потому плоским, правым $\left\langle X_{2}, \ldots, X_{n}\right\rangle$-модулем.

ОПРЕДЕЛЕНИЕ 3.6. Если $S$ - модуль над алгеброй $R$, то через $L(S)$ обозначается решетка подмодулей в $S$.

СлеДСТВИЕ 3.7. Пусть в $G$ выбран базис $X_{1}, \ldots, X_{n} u B=\left\langle X_{2}, \ldots, X_{n}\right\rangle$. Предположим, что $M$ - периодический конечнопорожденный B-модуль. Тогда сопоставление

$$
\varphi: L(M) \rightarrow L\left(A \otimes_{B} M\right), \quad \varphi(N)=A \otimes_{B} N,
$$

задает влохсение решеток.

ДокАЗАТЕЛьСтво. Покажем сначала, что $\varphi$ инъективно. Пусть $N, N^{\prime}$ - два $B$-подмодуля в $M$, причем

$$
A \otimes_{B} N^{\prime}=A \otimes_{B} N .
$$

В этом случае $A \otimes_{B} N^{\prime}=\left(A \otimes_{B} N^{\prime}\right)+\left(A \otimes_{B} N\right)=A \otimes_{B}\left(N+N^{\prime}\right)$. Таким образом, можно считать, что $N^{\prime} \subseteq N$.

Предположим, что $N \neq N^{\prime}$. В этом случае $N^{\prime \prime}=N / N^{\prime} \neq 0$ и по предложению 3.5 $A \otimes_{B} N^{\prime \prime} \neq 0$. С другой стороны, из (11)

$$
A \otimes_{B} N^{\prime \prime}=A \otimes_{B}\left(N / N^{\prime}\right) \simeq\left(A \otimes_{B} N\right) /\left(A \otimes_{B} N^{\prime}\right)=0 .
$$

Итак, отображение $\varphi$ является вложением.

Так как $A$ является проективным правым $B$-модулем, то по [11, с. $31-32]$

$$
A \otimes_{B}\left(N \cap N^{\prime}\right)=\left(A \otimes_{B} N\right) \cap\left(A \otimes_{B} N^{\prime}\right)
$$

для любых $B$-подмодулей $N, N^{\prime}$ в $M$. Кроме того,

$$
A \otimes_{B}\left(N+N^{\prime}\right)=\left(A \otimes_{B} N\right)+\left(A \otimes_{B} N^{\prime}\right) .
$$

Отметим в связи с последним следствием утверждение, по-существу доказанное в следствии 4.8 из [12]. 
ПРЕДЛОЖЕНИЕ 3.8. Если $G, X_{1}, \ldots, X_{n}, B$ как и в следствии $3.7, M$ - неприводимый $B$-модуль, то $A$-модуль $A \otimes_{B} M$ неприводим.

ДоказАТЕЛЬСтво. Так как $B$-модуль $M$ неприводим, то он конечнопорожден и по теореме 1.2 периодичен. Следовательно, $A$-модуль $A \otimes_{B} M$ конечнопорожден и периодичен. В частности, он артинов и по следствию 4.8 из [12] он неприводим.

Московский государственный университет

Поступило

им. М.В. Ломоносова

01.06 .95

E-mail: artamon@nw.math.msu.su

\section{СПИСОК ЦИТИРОВАННОЙ ЛИТЕРАТУРЫ}

[1] Артамонов В. А. Проективные модули над квантовыми алгебрами полиномов // Матем. сб. 1994. Т. 185. № 7. С. 3-12.

[2] Артамонов В. А. Модули над квантовыми полиномами // Матем. заметки. 1996. Т. 59. № 4. C. 497-503.

[3] Passman D. Infinite crossed products. San Diego: Acad. Press, 1989.

[4] Alev J., Chamarie M. Dérivations et automorphismes de quelques algèbres quantiques // Comm. Algebra. 1992. V. 20. №6. P. 1787-1802.

[5] Jordan D. A. Krull and global dimensions of certain iterated skew polynomial rings // Contemp. Math. 1992. V. 130. P. 201-213.

[6] Osborn M. J., Passman D.S. Derivations of skew polynomial rings // J. Algebra. 1995. V. 176. P. 417-448.

[7] Демидов Е. Е. О некоторых аспектах теории квантовых групп // УМН. 1993. Т. 48. №6. C. $39-74$.

[8] Montgomery S. Hopf algebras and their actions on rings. Conference Board of Math. Soc. V. 82. Providence: AMS, 1993.

[9] McConnell J. C., Pettit J. J. Crossed products and multiplicative analogues of Weyl algebras // J. London Math. Soc. 1988. V. 38. № 1. P. 47-55.

[10] Lam T. Y. Serre's conjecture. Lecture Notes in Math. V. 635. Berlin: Springer, 1978.

[11] Бурбаки Н. Коммутативная алгебра. М.: Мир, 1971.

[12] Hodges T. J., McConnell J. C. On Ore and skew-Laurent extensions of Noetherian rings // J. Algebra. 1981. V. 73. №1. P. 56-64. 